\title{
Article \\ On centre properties of irreducible subalgebras of compact elementary operators
}

\author{
W. Kangogo ${ }^{1, *}$, N. B. Okelo ${ }^{1}$ and O. Ongati ${ }^{1}$ \\ 1 Department of Pure and Applied Mathematics, Jaramogi Oginga Odinga, University of Science and Technology, Box \\ 210-40601, Bondo-Kenya.
}

Received: 12 August 2020; Accepted: 27 August 2020; Published: 31 August 2020.

\begin{abstract}
In this paper, we characterize the centre of dense irreducible subalgebras of compact elementary operators that are spectrally bounded. We show that the centre is a unital, irreducible and commutative $C^{*}$-subalgebra. Furthermore, the supports from the centre are orthogonal and the intersection of a nonzero ideal with the centre is non-zero.
\end{abstract}

Keywords: Centre, elementary operators, spectrally bounded.

MSC: 47B47, 47A30.

\section{Introduction}

$\mathbf{T}$ he class of elementary operators is among the classes of bounded linear operators on $C^{*}$-algebras. Elementary operators have great applications in operator theory, non-commutative algebraic geometry and solid state physics [1]. Moreover, several properties of the norm of elementary operators have been studied in [2-4] and [5]. Shulman and Turovskii [6] presented tensor spectral radius technique and tensor Jacobson radical which have applications to spectral theory of elementary operators and multiplication operators on Banach algebras. In [6], it was shown that the intersection of two flexible ideals is a flexible ideal with respect to the normed ideal. In the study of locally elementary operators, Nair [7] showed that every locally elementary operator is an elementary operator and every locally elementary operator is the strong limit of a sequence of elementary operators. Some properties of elementary operators have been studied like the coefficients and spectra [8]. Boudi and Bracic [8] characterized the relationship between non-invertibility (invertibility) of elementary operators and properties defining the coefficients.

In [8], it was proved that an operator $\Phi \in B(B(H))$ is non-invertible if it is a right zero divisor or left zero divisor and if a length 2 elementary operator $\Phi$ is annihilated by elementary operator, then there exists a multiplication operator $M$ such that $M \Phi=0$ (or $\Phi M=0$ ). Hejazian and Rostamani [9] proved that the class of spectrally compact operators is strictly contained in the class of compact operators and the set of spectrally compact operators on spectrally normed space $E$ is a right ideal of spectrally bounded operators which are two sided ideals. Moreover, Kittaneh [10] proved several spectral radii inequalities for sum, products and commutators of Hilbert operators and found that the spectral radius preserves commutativity by the following equation $r(A B)=r(B A)$ for every $A, B \in B(H)$.

Moreover, Okelo and Mogotu [11] established orthogonality and norm inequalities for commutators of derivations. In [12], a ring $R$ is said to be prime if it contains no non-zero orthogonal ideals. Mathieu [12] discussed the interrelations between primeness and properties of multiplications on prime $C^{*}$-algebras while in [13] the necessary and sufficient conditions for elementary operator $T_{A_{i}, B_{i}}(X)=A_{1} X B_{1}+\ldots+A_{n} X B_{n}$ to be identically zero or to compact map or (Hilbert space) for induced mapping on the Calkin algebra to be identically equal to zero were discussed. Also, Gogic and Timoney [14] established closure conditions of multiplication operators on $C^{*}$-algebras. It was shown in [14] that a basic elementary on a $C^{*}$-algebra $\mathcal{A}$ with the coefficients in $\mathcal{A}$ is norm closed for all primitive $C^{*}$-algebra $\operatorname{Prim} \mathcal{A}$ (where $\operatorname{Prim} \mathcal{A}$ is the primitive spectrum which is the set of irreducible representations of $\mathcal{A}$ equipped with Jacobson topology). Still on prime ideals, the authors [2] established the relationship between inner derivations implemented by a norm attainable element of a $C^{*}$-algebra to those of ideals and primitive ideals. 
Kumar and Rajpal [15] showed that the Banach projective tensor $\mathcal{A} \otimes_{\gamma} \mathcal{B}$ and operator space projective tensor product $\mathcal{A} \widehat{\otimes} \mathcal{B}$ of two $C^{*}$-algebras $\mathcal{A}$ and $\mathcal{B}$ are symmetric. Furthermore, in [15] the author showed that a Banach algebra is said to be quasi-central if no primitive ideal contain its centre. For a weakly Wiener Banach algebra $\mathcal{A}$ has an approximate identity element which is quasi-central that belongs to $\mathcal{Z}(\mathcal{A})$ [15]. In addition, studies on centrality and spectrum of algebra was shown [16], that semi-simple algebras containing some algebraic element whose centralizer is semi-perfect are Artinian and a semi-simple complex Banach algebra containing some element whose centralizer is algebraic are finite dimensional. Bratteli [17] proved that any separable abelian $C^{*}$-algebra is the centre of $C^{*}$-algebra with inductive limit of an increasing sequence of finite dimensional $C^{*}$-algebras.

Suppose that $E$ is a Banach lattice then its ideal centre $Z(E)$ is embedded naturally in the ideal centre $Z\left(E^{\prime}\right)$ of its dual. The embedding may be extended to a contractive algebra and lattice homomorphism of $Z(E)^{\prime \prime}$ into $Z\left(E^{\prime}\right)$. Orhon [18] showed that the extension is onto $Z\left(E^{\prime}\right)$ if and only if $E$ has a topological full centre. Other studies on centre are outlined in [1], for example, the centre of $C^{*}$-algebra $\mathcal{A}$ contains information about properties of operators defined on $\mathcal{A}$ which are compatible with ideals of $\mathcal{A}$ such as derivations, automorphisms, elementary operators, among others. In [1], it was shown that every $C^{*}$-algebra $\mathcal{B}$ of $\operatorname{Mloc}(\mathcal{A})$ is containing both $C_{b}$ and $\mathcal{A}$ is boundedly centrally closed. Sarsour and As'ad [19] established the relationship between centrality of Banach algebras and centrality of its closed subalgebras. In [19], it was shown that for a closed subalgebra $\mathcal{B}$ of a unital complex Banach algebra $\mathcal{A}$, then the quasicentral $Q(\mathcal{A}), \sigma$-quasicentral $Q_{\sigma}(\mathcal{A})$ and $\rho$-quasicentral $Q_{\rho}(\mathcal{A})$ sets need not be subsets of $Q(\mathcal{B}), Q_{\sigma}(\mathcal{B})$ and $Q_{\rho}(\mathcal{B})$ respectively. Moreover, $Q(\mathcal{B}), Q_{\sigma}(\mathcal{B})$ and $Q_{\rho}(\mathcal{B})$ need not be subsets of $Q(\mathcal{A}), Q_{\sigma}(\mathcal{A})$ and $Q_{\rho}(\mathcal{A})$ respectively. In addition, As'ad [20] studied the extended centre, extended quasi-centre, the extended $\sigma$-quasi centre and extended $\rho$-quasi centre of complex Banach algebra. In [20], if $\mathcal{A}$ is a unital complex Banach algebra then $Z_{e}(\mathcal{A}) \subseteq Q_{e} \subseteq Q_{\rho e}(\mathcal{A})$ and $Q_{e}(\mathcal{A}) \subseteq Q_{\rho e}(\mathcal{A}), Q_{e}(\mathcal{A}) \subseteq Q_{\sigma}(\mathcal{A})$ and $\mathcal{Z}_{e}(\mathcal{A})$ is a unital normed subalgebra of $\mathcal{A}, \mathcal{Z}(\mathcal{A}) \subseteq \mathcal{Z}_{e}(\mathcal{A}), Q(\mathcal{A}) \subseteq Q_{e}(\mathcal{A}), Q_{\sigma}(\mathcal{A}) \subseteq Q_{\sigma e}(\mathcal{A})$ and $Q_{\rho}(\mathcal{A}) \subseteq Q_{\rho e}(\mathcal{A})$. Furthermore, Rennison [21] gave a number of conditions related to centrality of Banach algebras which include non-unital algebras, analytic functions of quasi central elements and algebras having all quasi central elements are central.

We see that there are a lot of studies on elementary operators, locally elementary operators, spectrum, spectral radius, compactness, commutativity and tensor products of Banach algebras. However, properties of the centre of dense irreducible subalgebras of compact elementary operators that are spectrally bounded remain interesting. Therefore, in this paper we endeavour to investigate properties of the centre of dense irreducible subalgebras of compact elementary operators that are spectrally bounded on $C^{*}$-algebras.

\section{Preliminaries}

In this section, we outline preliminary concepts which are useful in the sequel. Let $\mathcal{A}$ be a Banach algebra. We denote a dense irreducible $C^{*}$-subalgebra of $\mathcal{A}$ by $\mathcal{A}_{\mathcal{D I R}}$. The algebra of all compact elementary operators on $\mathcal{A}$ is denoted by $\mathcal{C}(\mathcal{E})$. We also denote the algebra of all spectrally bounded compact elementary operators on $\mathcal{A}_{\mathcal{D I R}}$ is denoted by $\mathcal{C}_{\mathcal{S B D}}(\mathcal{E})$.

Definition 1. ([22]) Let $V$ be a linear vector space. A non-negative real valued function $\|\|:. V \rightarrow \mathbb{R}$ is called a norm on $V$ if it satisfies the following conditions:

(i). $\|a\| \geq 0$ and $\|a\|=0$, if and only if $a=0$, for all $a \in V$.

(ii). $\|\alpha a\|=|\alpha|\|a\|$, for all $a \in V$ and $\alpha \in \mathbb{K}$.

(iii). $\|a+b\| \leq\|a\|+\|b\|$, for all $a, b \in V$.

The ordered pair $(V,\|\|$.$) is called a normed space.$

Definition 2. ([23], Section 2) Consider a $C^{*}$-algebra $\mathcal{A}$ and let $T: \mathcal{A} \rightarrow \mathcal{A}$. The operator $T$ is called an elementary operator if it has the following representation: $T_{A_{i}, B_{i}}(X)=\sum_{i=1}^{n} A_{i} X B_{i} \forall A_{i}, B_{i}$ are fixed in $\mathcal{A}$ or $\mathcal{M}(\mathcal{A})$ is multiplier algebra of $\mathcal{A}$. For $A, B \in B(H)$, we define particular elementary operators

(i). the left multiplication operator $L_{A}: B(H) \rightarrow B(H)$ by $L_{A}(X)=A X, \forall X \in B(H)$.

(ii). the right multiplication operator $R_{B}: B(H) \rightarrow B(H)$ by $R_{B}(X)=X B, \forall X \in B(H)$.

(iii). the generalized derivation (implemented by $A, B)$ by $\delta_{A, B}(X)=L_{A}-R_{B}, \forall X \in B(H)$.

(iv). the basic elementary operator (implemented by $A, B$ ) by $M_{A, B}(X)=A X B, \forall X \in B(H)$. 
(v). The Jordan elementary operator (implemented by $A, B)$ by $\mathfrak{U} A, B(X)=A X B+B X A, \forall X \in B(H)$.

Definition 3. ([24], Definition 4.1) If $\mathcal{A}$ is any $C^{*}$-algebra and $\Phi: \mathcal{A} \rightarrow B(H)$ is a representation then $\Phi$ is irreducible if $\Phi(\mathcal{A})$ is an irreducible subalgebra of $B(H)$.

Definition 4. ([25], Definition 2.2) Let $\mathcal{A}$ and $\mathcal{B}$ be complex Banach algebras. A linear mapping $T: \mathcal{A} \rightarrow \mathcal{B}$ is called spectrally bounded if there exists a constant $M \geq 0$ such that $r(T x) \leq M r(x)$ and spectrally infinitesimal if $r(T(x))=0$, for all $x \in \mathcal{A}$. If $r(T(x))=r(x)$, for all $x \in \mathcal{A}$ we say that $T$ is a spectral isometry. If $r(x)=0$, then $x$ is called quasi-nilpotent.

Definition 5. ([26], Definition 4.2.12) A representation $T$ of an algebra $\mathcal{A}$ on a linear space $X$ is called strictly dense if whenever $x_{1}, x_{2}, \ldots, x_{n}$ is a finite list of linearly independent vectors in $X$ and $y_{1}, y_{2}, \ldots, y_{n}$ is a list of vectors in $X$ then there is an element $a \in A$ with $T_{a} x_{j}=y_{j}$ for $j=1,2, \ldots, n$.

Definition 6. Let $T_{A_{i}, B_{i}} \in \mathcal{C}(\mathcal{E})$. We define the spectral operator norm of $T_{A_{i}, B_{i}}$ by $\left\|T_{A_{i}, B_{i}}\right\|_{\sigma}=\inf \{M \geq$ $\left.0\left|r\left(T_{A_{i}, B_{i}}\left(X_{n}\right)\right)\right| \leq M r(X)\right\}$, for all $X \in \mathcal{A}_{\mathcal{D I} \mathcal{R}}$.

Definition 7. Let $\mathcal{C}_{\mathcal{S B D}}(\mathcal{E})$ be a Jordan Banach algebra. Then two elements $P, Q \in \mathcal{C}_{\mathcal{S B D}}(E)$ are orthogonal if one the following equivalent conditions hold:

(i). $P \leq I-Q$.

(ii). $P \circ Q=0$.

(iii). $\mathfrak{U}_{P} \mathfrak{U}_{Q}=0$.

Definition 8. ([27], Definition 1.3) The centre of a Banach algebra $\mathcal{A}$ is defined by $\mathcal{Z}(\mathcal{A})=\{a \in \mathcal{A}: a x=$ $x a, \forall x \in \mathcal{A}\}$.

Definition 9. ([28], Section 3) A Jordan homomorphism between two Banach algebras $\mathcal{A}$ and $\mathcal{B}$ is a linear mapping $T: \mathcal{A} \rightarrow \mathcal{B}$ which preserves the derived Jordan product, i.e $T(a b+b a)=T(a) T(b)+$ $T(b) T(a), \forall a, b \in \mathcal{A}$.

Definition 10. ([29]) A Jordan-Banach algebra $\mathcal{A}$ is a Jordan algebra with the complete norm $\|x \circ y\| \leq\|x\|\|y\|$, for all $x, y \in \mathcal{A}$.

Definition 11. ([26], Definition 4.1.1) Let $\mathcal{A}$ be an algebra and let $X$ be a linear space. A representation (anti-representation) $T$ of $\mathcal{A}$ on $X$ is a homomorphism (anti-homomorphism) $a \mapsto T_{a}$ of $\mathcal{A}$ in $B(X)$. $\mathrm{A}$ subspace $Y$ of $X$ is said to be $T$-invariant if $T_{a} y$ belong to $Y$, for all $y \in Y$ and $a \in \mathcal{A}$. The representation (anti-representation) $T$ of $\mathcal{A}$ on $X$ is said to be:

(i). Faithful if the homomorphism is injective.

(ii). Trivial if $T a=0$ for every $a \in \mathcal{A}$.

(iii). Irreducible if $\{0\}$ and $X$ are the only $T$-invariant subspaces and $T$ is not trivial.

(iv). Cyclic if there exists a vector $z \in X$ satisfying $X=\left\{T_{a} z: a \in \mathcal{A}\right\}$.

Definition 12. ([30], Definition 2.1.2) If $E$ is a vector space, a set $A \subset E$ is called convex if $x, y \in A, t \in$ $[0,1] \Longrightarrow t x+(1-t) y \in A$.

Definition 13. ([31], Definition 3.10) Let $X$ be a linear space and $C$ be a convex subset of $X$. A point $x \in C$ is said to be extreme point of $C$ if and only if $C \backslash\{x\}$ is still convex. That is, if any time $x=\lambda x_{1}+(1-\lambda) x_{2}$ where $x_{1}, x_{2} \in C$ and $0<\lambda<1$, then $x=x_{1}=x_{2}$.

\section{Main results}

In this section, we give results on the centre of dense irreducible subalgebras of compact elementary operators that are spectrally bounded. 
Proposition 1. The centre $Z\left[\mathcal{C}_{\mathcal{S B D}}(\mathcal{E})\right]$ is an irreducible subalgebra of $\mathcal{C}_{\mathcal{S B D}}(\mathcal{E})$.

Proof. The centre $Z\left[\mathcal{C}_{\mathcal{S B D}}(\mathcal{E})\right]=\left\{S \in \mathcal{C}_{\mathcal{S B D}}(\mathcal{E}) ; S T=T S, \forall T \in \mathcal{C}_{\mathcal{S B D}}(\mathcal{E})\right\}$. To show that $Z\left[\mathcal{C}_{\mathcal{S B D}}(\mathcal{E})\right]$ is indeed a subalgebra of $\mathcal{C}_{\mathcal{S B D}}(\mathcal{E})$, let $Q \in \mathcal{C}_{\mathcal{S B D}}(\mathcal{E})$ then for all $S, T \in Z\left[\mathcal{C}_{\mathcal{S B D}}(\mathcal{E})\right]$, we have $S Q=Q S$ and $T Q=Q T$. Also $(S-T) Q=S Q-T Q=Q S-Q T=Q(S-T)$ and $(S+T) Q=S Q+T Q=Q S+$ $Q T=Q(S+T)$. Similarly, $(S T) Q=S(T Q)=S(Q T)=(S Q) T=(Q S) T=Q(S T)$. Finally, we show that $Z\left[\mathcal{C}_{\mathcal{S B D}}(\mathcal{E})\right]$ is irreducible, if $T \in Z\left[\mathcal{C}_{\mathcal{S B D}}(\mathcal{E})\right]$ is a projection i.e $T^{2}=T$ and $T S=S T \Rightarrow S=\{0\}$ and $T=I$ hence $Z\left[\mathcal{C}_{\mathcal{S B D}}(\mathcal{E})\right]$ is an irreducible subalgebra of $\mathcal{C}_{\mathcal{S B D}}(\mathcal{E})$.

Proposition 2. The centre $Z\left[\mathcal{C}_{\mathcal{S B D}}(\mathcal{E})\right]$ is a commutative irreducible subalgebra of $\mathcal{C}_{\mathcal{S B D}}(\mathcal{E})$.

Proof. From Definition 8, the centre $Z\left[\mathcal{C}_{\mathcal{S B D}}(\mathcal{E})\right]$ is commutative. Now, let $S, T \in Z\left[\mathcal{C}_{\mathcal{S B D}}(\mathcal{E})\right]$ then for all $R \in Z\left[\mathcal{C}_{\mathcal{S B D}}(\mathcal{E})\right]$ we have $S R=R S$ and $T R=T R$. Hence, $R(S-T)=R S-R T=S R-T R=R(S-T)$ and $R(S+T)=R S+R T=S R+T R=R(S+T)$ hold. Furthermore, $R(S T)=(R S) T=(S R) T=S(R T)=$ $S(T R)=(S T) R$ which implies that $S T \in Z\left[\mathcal{C}_{\mathcal{S B D}}(\mathcal{E})\right]$. Finally, we show that $Z\left[\mathcal{C}_{\mathcal{S B D}}(\mathcal{E})\right]$ is irreducible, if $T \in Z\left[\mathcal{C}_{\mathcal{S B D}}(\mathcal{E})\right]$ is a projection then the only trivial projection is $T^{2}=T$ and $T S=S T \Rightarrow S=\{0\}$ and $T=I$ hence $Z\left[\mathcal{C}_{\mathcal{S B D}}(\mathcal{E})\right]$ is a commutative irreducible subalgebra of $\mathcal{C}_{\mathcal{S B D}}(\mathcal{E})$.

Proposition 3. Let $\mathcal{C}_{\mathcal{S B D}}(\mathcal{E})$ be unital $C^{*}$-algebra. Then $Z\left[\mathcal{C}_{\mathcal{S B D}}(\mathcal{E})\right]$ is a unital $C^{*}$-subalgebra of $\mathcal{C}_{\mathcal{S B D}}(\mathcal{E})$.

Proof. Consider a positive cone $E$ and $F$ a unit ball in $Z\left[\mathcal{C}_{\mathcal{S B D}}(\mathcal{E})\right]$ such that the set $G=E \cap F$ contains extreme points of the centre $Z\left[\mathcal{C}_{\mathcal{S B D}}(\mathcal{E})\right]$ which are unital. We proceed to show that the extreme points of the convex set $G$ denoted by $\delta_{e}(G)$ coincides with the the set of idempotents in $Z\left[\mathcal{C}_{\mathcal{S B D}}(\mathcal{E})\right]$ which are unital. Now, let $P$ be a projection such that $P=\frac{S+T}{2} ; S, T \in G=E \cap F$. Then $S=2 P-T$ since $0 \leq S \leq 2 P, S=P Z\left[\mathcal{C}_{\mathcal{S B D}}(\mathcal{E})\right] P$ and $S$ commutes with $T$ from Proposition 2. Therefore, the centre $Z\left[\mathcal{C}_{\mathcal{S B D}}(\mathcal{E})\right]$ is a commutative $C^{*}$-subalgebra generated by $S$ and $T$ are equal hence $S=T=P$. Furthermore, the centre $Z\left[\mathcal{C}_{\mathcal{S B D}}(\mathcal{E})\right]$ is a spectral dual and its unit ball $F$ is weak*-compact and applying Krein-Milman theorem, $F$ must have an extreme point $S$. So applying spectral decomposition, $S=S^{+}-S^{-}$where $S^{+}, S^{-}$are elements $\mathcal{C}_{\mathcal{S B D}}(\mathcal{E})$ corresponding to functions on the spectrum of $S, \sigma(S)$, defined by

$\Psi^{+}(\lambda)=\left\{\begin{array}{ll}\lambda, & \lambda \geq 0 ; \\ 0, & \lambda \leq 0 .\end{array}\right.$ and $\Psi^{-}(\lambda)=\left\{\begin{array}{ll}0, & \lambda \geq 0 ; \\ -\lambda, & \lambda \leq 0 .\end{array}\right.$ which are closed under algebraic multiplicity since the norm $\left\|S^{2}-T^{2}\right\|=\max \left\{\|S\|_{2},\|T\|_{2}\right\}$. Consider the self-adjoint elements in $Z\left[\mathcal{C}_{\mathcal{S B D}}(\mathcal{E})\right]$ which are unital such that $S=S^{+}-S^{-}$is an extreme point in $\delta_{e}\left(Z\left[\mathcal{C}_{\mathcal{S B D}}(\mathcal{E})\right]^{*} \cap F\right)$, where $\delta_{e}($.$) is extreme point. It follows that$ $S^{+}$and $S^{-}$must be extreme in $E \cap F$. For instance, if $S^{+}=\frac{X+Y}{2}$, for all $X, Y \in E \cap F$ then $X-S^{-}, Y-S^{-} \in$ $Z\left[\mathcal{C}_{\mathcal{S B D}}(\mathcal{E})\right]^{*} \cap F$ and $S=\frac{X-S^{-}+Y-S^{-}}{2}$. Therefore, $S=X-S^{-}=Y-S^{-}$and so $X=Y=S^{+}$. Following the above procedure, $S^{-}$is an extreme point in $E \cap F$. Since $S^{+}$and $S^{-}$are projections, then $S^{+}+S^{-}=I$ and $S^{2}=P$. Also, let $T$ be an element of $\left(I-2 \mathfrak{L}_{P}+\mathfrak{U}_{P}\right) Z\left[\mathcal{C}_{\mathcal{B B D}}(\mathcal{E})\right]$ such that $\|T\| \leq 1$ and $R \in Z\left[\mathcal{C}_{\mathcal{S B D}}(\mathcal{E})\right]$ then $T=\left(I-2 \mathfrak{L}_{P}+\mathfrak{U}_{P}\right) R$ is a Jordan Banach algebra isomorphism. Therefore, the subalgebra $Z[S, R]$ of $Z\left[\mathcal{C}_{\mathcal{S B D}}(\mathcal{E})\right]$ generated by $S$ and $R$ is isometrically Jordan isomorphic to a Jordan Banach algebra of self-adjoint operators on a complex Hilbert space. Thus,

$$
S \circ T=0
$$

and

$$
S^{2} \circ T^{2}=0 .
$$

It follows from Equation 1 that $P \circ S=0$ and Equation 2 that $\mathfrak{U}_{P} T^{2}=0$ since $\left\{P T^{2} P\right\}=0$ and by Jordan isomorphism property, we have

$$
\left\|P+T^{2}\right\|^{\frac{1}{2}}=\max \left\{1,\left\|T^{2}\right\|^{\frac{1}{2}}\right\} \leq 1 .
$$

Applying Equation 1 and Equation 2 we have,

$$
\|S \pm T\|=\left\|(S \pm T)^{2}\right\|^{\frac{1}{2}}=\left\|S^{2} \pm 2(S \circ T)+T^{2}\right\|=\left\|P+T^{2}\right\|^{\frac{1}{2}} \leq 1 .
$$


However, we know that $S$ is an extreme point of $F$ and $S=\frac{1}{2}(S+T)+\frac{1}{2}(S-T)$. So, $T=0$ and $\left(I-2 \mathfrak{L}_{P}+\right.$ $\left.\mathfrak{U}_{P}\right) Z\left[\mathcal{C}_{\mathcal{S B D}}(\mathcal{E})\right]=\{0\}$. This shows that for all $W \in Z\left[\mathcal{C}_{\mathcal{S B D}}(\mathcal{E})\right],\|W-P \circ W\|^{2} \leq\left\|\left(I-2 \mathfrak{L}_{P}+Q\right) W\right\|\|W\|=$ 0 and linearity follows since the projection $P$ is an identity for $Z\left[\mathcal{C}_{\mathcal{S B D}}(\mathcal{E})\right]$. Thus, $Z\left[\mathcal{C}_{\mathcal{S B D}}(\mathcal{E})\right]$ is a unital $C^{*}$-subalgebra of $\mathcal{C}_{\mathcal{S B D}}(\mathcal{E})$.

Theorem 1. Let $P$ and $Q$ be idempotents in $\mathcal{C}_{\mathcal{S B D}}(\mathcal{E})$. If $\mathcal{C}_{\mathcal{S B D}}(\mathcal{E})$ is a Jordan-Banach algebra with Identity I and $P \in Z\left[\mathcal{C}_{\mathcal{S B D}}(\mathcal{E})\right]$, then the supports from the centre $z(P)$ and $z(Q)$ are orthogonal.

Proof. Let $P \in \mathcal{C}_{\mathcal{S B D}}(\mathcal{E})$ then the support from the centre $z(P)$ is defined by $z(P)=\wedge\{Q: \quad Q \in$ $\left.Z\left[\mathcal{C}_{\mathcal{S B D}}(\mathcal{E})\right], P \leq Q\right\}$ or

$$
z(P)=\wedge\left\{Q: Q \in Z\left[\mathcal{C}_{\mathcal{S B D}}(\mathcal{E})\right], \mathfrak{U}_{Q} P=Q\right\} .
$$

If $W \in Z\left[\mathcal{C}_{\mathcal{S B D}}(\mathcal{E})\right]$ and $P \in \mathcal{C}_{\mathcal{S B D}}(\mathcal{E})$ with $P \leq Z$, then the support from the centre of $P$ in a Jordan-Banach algebra $\mathcal{C}_{\mathcal{S B D}}(\mathcal{E})$ is denoted by $z_{w}(P)$. In this case, the centre $Z\left[\mathcal{C}_{\mathcal{S B D}}(\mathcal{E})\right]$ of a Jordan Banach algebra $\mathcal{C}_{\mathcal{S B D}}(\mathcal{E})$ is an associative Jordan Banach algebra and is isomorphic to Jordan Banach algebra $K\left(\mathcal{H}_{P}, \mathbb{M}_{3}^{8}\right)$ (where $K\left(\mathcal{H}_{P}, \mathbb{M}_{3}^{8}\right)$ is Jordan Banach algebra of continuous functions from Hyperstonean space $\mathcal{H}_{P}$ into $\mathbb{M}_{3}^{8}$ hermitian $3 \times 3$ matrices over Cayley numbers). Therefore, the following conditions hold,

$$
\mathfrak{U}_{P}+\mathfrak{U}_{I-P}=I
$$

and

$$
\mathfrak{U}_{P} \mathfrak{U}_{Q}=\mathfrak{U}_{Q} \mathfrak{U}_{P}, \forall Q \in \mathcal{C}_{\mathcal{S B D}}(\mathcal{E}) .
$$

Thus, the centre $Z\left[\mathcal{C}_{\mathcal{S B D}}(\mathcal{E})\right]$ is equal to $K\left(\mathcal{H}_{P}\right)$, the dual space of real valued continuous functions on $\mathcal{H}_{P}$ and the support from the centre $z(P)$ is defined by

$$
\begin{aligned}
z(P) & =\wedge\left\{\chi_{A}: A \subseteq \mathcal{H}_{P}, \text { closed and open, } P(E) \leq \chi_{A}(E) I, \forall E \in \mathcal{H}_{P}\right\} \\
& =\wedge\left\{\chi_{A}: A \subseteq \mathcal{H}_{P}, \text { closed and open, } P(E)=0, \forall E \in A\right\} \\
& =\left(\vee\left\{\chi_{A}: A \subseteq \mathcal{H}_{P}, \text { closed and open, } P(E)=0, \forall E \in A\right\}\right)^{\prime} \\
& =\chi_{B}, B \text { is the complement of the closure of the set } B_{0} .
\end{aligned}
$$

Here $\chi_{A}$ is the characteristic function of $A \subseteq \mathcal{H}_{P}, I$ is the identity in $\mathbb{M}_{3}^{8}$ and $B$ is the complement of the closure of the set $B_{0}$, that is,

$$
B_{0}=\cup\left\{A: A \subseteq \mathcal{H}_{P} \text {, closed and open, } P(E)=0, \forall E \in A\right\} .
$$

The centre $z(Q)$ is defined by

$$
\begin{aligned}
z(Q) & =\wedge\left\{\chi_{A}: A \subseteq \mathcal{H}_{P}, \text { closed and open, } Q(E) \leq \chi_{A}(E) I, \forall E \in \mathcal{H}_{P}\right\} \\
& =\wedge\left\{\chi_{A}: A \subseteq \mathcal{H}_{P}, \text { closed and open, } Q(E)=0, \forall E \in A\right\} \\
& =\left(\vee\left\{\chi_{A}: A \subseteq \mathcal{H}_{P}, \text { closed and open, } Q(E)=0, \forall E \in A\right\}\right)^{\prime} \\
& =\chi_{D}, D \text { is the complement of the closure of the set } D_{0},
\end{aligned}
$$

Also, here $\chi_{D}$ is the characteristic function of $A \subseteq \mathcal{H}_{P}$ and $D$ is the complement of the closure of the set $D_{0}$ given by

$$
D_{0}=\cup\left\{A: A \subseteq \mathcal{H}_{P} \text {, closed and open, } Q(E)=0, \forall E \in A\right\} .
$$

Next, we need to show that $B \cap D$ is empty. Let $E \in B \cap D$, then $P(E)$ and $Q(E)$ are idempotents in $\mathbb{M}_{3}^{8}$ such that $\mathfrak{U}_{P(E), Q(E)} \mathbb{M}_{3}^{8}=\{0\}$. Thus, atleast one of $P(E)$ and $Q(E)$ is zero. Thus, $B_{1}$ and $D_{1}$ are closed subsets of closed and open set $B \cap D$ given by

$$
B_{1}=\{E: E \in B \cap D, P(E)=0\}, D_{1}=\{E: E \in B \cap D, Q(E)=0\},
$$


which covers $B \cap D$. Consequently, the open set $B_{1}^{\prime} \cap B \cap D$ is contained in closed set $B_{1}$. In fact, the closed and

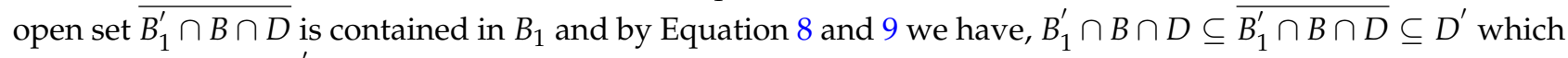
shows that the set $B_{1}^{\prime} \cap B \cap D$ is empty. Thus, the closed and open set $B \cap D$ is contained in the $B_{1}$ and from Equation 7, we have $B \cap D \subseteq B^{\prime}$ hence we see that $B \cap D$ is empty. Therefore, we conclude that the supports from the centres $z(P)$ and $z(Q)$ are orthogonal.

Corollary 2. Let $P$ be an idempotent in a Jordan-Banach algebra $\mathcal{C}_{\mathcal{S B D}}(\mathcal{E})$. Then the centre $Z\left[\mathcal{C}_{\mathcal{S B D}}^{\diamond}(\mathcal{E})\right]$ of hereditary Jordan-Banach subalgebra $\mathcal{C}_{\mathcal{S B D}}^{\diamond}(\mathcal{E})$ of $\mathcal{C}_{\mathcal{S B D}}(\mathcal{E})$ coincides with the range of the centre $\mathfrak{U}\left(Z\left[\mathcal{C}_{\mathcal{S B D}}(\mathcal{E})\right]\right)$ of $\mathcal{C}_{\mathcal{S B D}}(\mathcal{E})$ under the projection $\mathfrak{U}_{P}$.

Proof. We know that $\mathcal{C}_{\mathcal{S B D}}(\mathcal{E})$ is an associative Jordan-Banach algebra. It follows that the centres $Z\left[\mathcal{C}_{\mathcal{S B D}}(\mathcal{E})\right]$ and $Z\left[\mathcal{C}_{\mathcal{S} B \mathcal{B}}^{\diamond}(\mathcal{E})\right]$ are associative Jordan Banach subalgebras which are idempotent. We prove that the projection $\mathfrak{U}_{P}$ maps idempotents $\mathfrak{U}\left(Z\left[\mathcal{C}_{\mathcal{S B D}}(\mathcal{E})\right]\right)$ onto $\mathfrak{U}\left(Z\left[\mathcal{C}_{\mathcal{S B D}}^{\diamond}(\mathcal{E})\right]\right)$. Thus, for every $Q \in \mathfrak{U}\left(Z\left[\mathcal{C}_{\mathcal{S B D}}(\mathcal{E})\right]\right)$ and using Equation 6, $\left(\mathfrak{U}_{P} Q\right)^{2}=\mathfrak{U}_{P} \mathfrak{U}_{Q} P^{2}=\mathfrak{U}_{P} \mathfrak{U}_{Q} \mathfrak{U}_{P} I=\mathfrak{U}_{P} \mathfrak{U}_{P} \mathfrak{U}_{Q} I=\mathfrak{U}_{P} Q$. Thus, $\mathfrak{U}_{P} Q \in \mathfrak{U}\left(\mathcal{C}_{\mathcal{S B D}}(\mathcal{E})\right)$. Moreover, using Equation5 and Equation 6, we have

$$
\begin{aligned}
\mathfrak{U}_{\mathfrak{U}_{P} Q}+\mathfrak{U}_{P-\mathfrak{U}_{P} Q} & =\mathfrak{U}_{\mathfrak{U}_{P} Q}+\mathfrak{U}_{\mathfrak{U}_{P}(I-Q)} \\
& =\mathfrak{U}_{P} \mathfrak{U}_{Q} \mathfrak{U}_{P}+\mathfrak{U}_{P} \mathfrak{U}_{I-Q} \mathfrak{U}_{P} \\
& =\mathfrak{U}_{P}\left(\mathfrak{U}_{Q}+\mathfrak{U}_{I-Q}\right)=\mathfrak{U}_{P} .
\end{aligned}
$$

This shows that $\mathfrak{U}_{P} Q$ is central idempotent in $\mathfrak{U}\left(\mathcal{C}_{\mathcal{S B D}}(\mathcal{E})\right)$. We need to show that the for every central idempotent $R \in \mathcal{C}_{\mathcal{S B D}}^{\diamond}(\mathcal{E})$, there exists a central idempotent $Q \in \mathcal{C}_{\mathcal{S B D}}(\mathcal{E})$ with range $R$ under projection $\mathfrak{U}_{P}$. So we show that $R$ and $P-R$ are idempotents in $\mathcal{C}_{\mathcal{S B D}}(\mathcal{E})$ and applying Equation 5 , we have $\mathfrak{U}_{R} \mathfrak{U}_{P}+\mathfrak{U}_{P-R} \mathfrak{U}_{P}=$ $\mathfrak{U}_{P}$. Moreover, $R, P-R \leq P$, hence

$$
\mathfrak{U}_{R}+\mathfrak{U}_{P-R}=\mathfrak{U}_{P},
$$

or $\mathfrak{U}_{R, P-R} \mathcal{C}_{\mathcal{S B D}}(\mathcal{E})=\{0\}$. It is known from Theorem 1 that the supports from the centre $z(R)$ and $Z(P-R)$ of the idempotents $R$ and $P-R$ are orthogonal. Hence, from Equation 6 we have

$$
\mathfrak{U}_{R} z(R)=\mathfrak{U}_{R} \mathfrak{U}_{z(R)} I=\mathfrak{U}_{z(R)} \mathfrak{U}_{R} I=\mathfrak{U}_{z(R)} R=R,
$$

and by Equation 5 we have

$$
\mathfrak{U}_{P-R} z(R)=\mathfrak{U}_{z(R)}(P-R)=(P-R)-\mathfrak{U}_{I-Z(R)}(P-R) .
$$

The orthogonality of the centres $z(P)$ and $z(P-R)$ implies that $z(P-R) \leq I-Z(R)$. Hence by Equation 4 and Equation 12

$$
\mathfrak{U}_{P-R} z(R)=0 .
$$

And applying Equation 10, Equation 11 and Equation13, we obtain the equation $\mathfrak{U}_{P} z(R)=\mathfrak{U}_{R} z(R)+$ $\mathfrak{U}_{P-R} Z(R)=R$. Thus, the centre $Z\left[\mathcal{C}_{\mathcal{S} B \mathcal{D}}^{\diamond}(\mathcal{E})\right]$ of hereditary Jordan-Banach subalgebra $\mathcal{C}_{\mathcal{S B D}}^{\diamond}(\mathcal{E})$ coincides with the range of the centre $\mathfrak{U}\left(Z\left[\mathcal{C}_{\mathcal{S B D}}(\mathcal{E})\right]\right)$ under the projection $\mathfrak{U}_{P}$.

Proposition 4. Let $\mathcal{C}_{\mathcal{S B D}}(\mathcal{E})$ be a prime and irreducible $C^{*}$-subalgebra with polynomial identity I and centre $Z\left[\mathcal{C}_{\mathcal{S B D}}(\mathcal{E})\right]$. If $\mathcal{J}$ is a nonzero ideal of $\mathcal{C}_{\mathcal{S B D}}(\mathcal{E})$ then $\mathcal{J} \cap Z\left[\mathcal{C}_{\mathcal{S B D}}(\mathcal{E})\right]$ is non-zero.

Proof. Let $\mathcal{A}_{\mathcal{S B D}} \subseteq \mathcal{C}_{\mathcal{S B D}}(\mathcal{E})$ be such that there exists a homomorphism from $\mathcal{C}_{\mathcal{S B D}}(\mathcal{E})$ onto $\mathcal{A}_{\mathcal{S B D}}$. Since $\mathcal{A}_{\mathcal{S B D}}$ is $C^{*}$-subalgebra of $\mathcal{C}_{\mathcal{S B D}}(\mathcal{E})$ then $\mathcal{A}_{\mathcal{S B D}}$ is prime and irreducible with the same polynomial identity $I$ and hence has the same dimension less than $\left(\frac{n}{2}\right)^{2}$ over its centre $Z\left[\mathcal{C}_{\mathcal{S B D}}(\mathcal{E})\right]_{i}$ by (Kaplansky's theorem). Let $\Phi_{i}: \mathcal{C}_{\mathcal{S B D}}(\mathcal{E}) \rightarrow \mathcal{A}_{\mathcal{S B D}}$ be a canonical projection such that $\bigcap_{i} \operatorname{Ker} \Phi_{i}=\{0\}$, whenever we restrict $\Phi_{i}$ to ideal $\mathcal{J}$ is nonzero for every $i$ and set $F=\left\{i: \Phi_{i} \mid \mathcal{J}\right.$ is nonzero $\}$. Suppose $\Phi_{i}: \mathcal{C}_{\mathcal{S B D}}(\mathcal{E}) \rightarrow \mathcal{A}_{\mathcal{S B D}}$ is surjective, then $\Phi_{i}(\mathcal{J})$ is a nonzero ideal of $\mathcal{A}_{\mathcal{S B D}}$ for every $i \in F$ and thus $\mathcal{A}_{\mathcal{S B D}}$ is prime and irreducible, since for every $i \in F, \Phi_{i}$ restricted to $\mathcal{J}$ is surjective. Suppose further that $i_{0} \in F$ such that $\mathcal{A}_{\mathcal{S B D}}^{0}$ has maximal dimension $n_{0}^{2}$ over its centre for every $i \in F$ and let $X_{n 0}$ be a non-vanishing central polynomial for any $\mathcal{A}_{\mathcal{S B D}}$ of degree $n$ over 
its centre $Z\left[\mathcal{C}_{\mathcal{S B D}}(\mathcal{E})\right]$. Since $X_{n 0}$ is central for $\mathbb{M}_{n 0}$, then $X_{n 0}$ is central for smaller degree matrix algebras, hence $X_{n 0}$ is central for $\mathcal{A}_{\mathcal{S B D}}$ for every $i \in F$. Also, Suppose that $A_{1}, . ., A_{m} \in \mathcal{A}_{\mathcal{S B D}}^{0}$ such that $X_{n 0}\left(A_{1}, \ldots, A_{m}\right) \neq 0$ and let $J_{1}, \ldots, J_{m} \in \mathcal{J}$ such that $\Phi_{i 0}\left(J_{k}\right)=A_{k}, 1 \leq k \leq m$. Then $X_{n 0}$ is homogenous of degree $>0$ and its constant term is 0 , for all $i \notin F$, so

$$
\Phi_{i}\left(X_{n 0}\left(J_{1}, \ldots, J_{m}\right)\right)=X_{n 0}\left(\Phi_{i}\left(J_{1}\right), \ldots, \Phi_{i}\left(J_{m}\right)\right)=X_{n 0}(0, \ldots, 0)=0
$$

and $\Phi_{i}\left(X_{n 0}\left(J_{1}, \ldots, J_{m}\right)\right)=X_{n 0}\left(\Phi_{i}\left(J_{1}\right), \ldots, \Phi_{i}\left(J_{m}\right)\right) \in Z\left[\mathcal{C}_{\mathcal{S B D}}(\mathcal{E})\right]_{i}$, for all $i \in F$. In particular,

$$
\Phi_{i_{0}}\left(X_{n 0}\left(J_{1}, \ldots, J_{m}\right)\right)=X_{n 0}\left(\Phi_{i_{0}}\left(J_{1}\right), \ldots, \Phi_{i_{0}}\left(J_{m}\right)\right)=X_{n 0}\left(A_{1}, \ldots, A_{m}\right) \neq 0
$$

but belong $Z\left[\mathcal{C}_{\mathcal{S B D}}(\mathcal{E})\right]_{i_{0}}$. Hence $X_{n 0}\left(J_{1}, \ldots, J_{m}\right) \in Z\left[\mathcal{C}_{\mathcal{S B D}}(\mathcal{E})\right]$ and is non-zero. However, $X_{n 0}\left(J_{1}, \ldots, J_{m}\right) \in \mathcal{J}$, hence $\mathcal{J} \cap Z\left[\mathcal{C}_{\mathcal{S B D}}(\mathcal{E})\right]$ is non-zero.

Lemma 1. Let $\mathcal{C}_{\mathcal{S B D}}(\mathcal{E})$ be a prime and irreducible $C^{*}$-subalgebra with polynomial identity I and centre $Z\left[\mathcal{C}_{\mathcal{S B D}}(\mathcal{E})\right]$. Then $\mathcal{C}_{\mathcal{S B D}}(\mathcal{E})$ is simple.

Proof. From Proposition $4, \mathcal{J}$ is a nonzero ideal of $\mathcal{C}_{\mathcal{S B D}}(\mathcal{E})$ and $\mathcal{J} \cap Z\left[\mathcal{C}_{\mathcal{S B D}}(\mathcal{E})\right]$ is non-zero. Thus, it follows that $\mathcal{J}=\mathcal{C}_{\mathcal{S B D}}(\mathcal{E})$ is simple.

Theorem 3. Let $\mathcal{C}_{\mathcal{S B D}}(\mathcal{E})$ be a prime and irreducible $C^{*}$-subalgebra with polynomial identity and centre $Z\left[\mathcal{C}_{\mathcal{S B D}}(\mathcal{E})\right]$. Then there exists simple prime $C^{*}$-subalgebra $\mathcal{B}_{\mathcal{S B D}}$ equal to $\mathcal{C}_{\mathcal{S B D}}(\mathcal{E}) \overline{Z\left[\mathcal{C}_{\mathcal{S B D}}(\mathcal{E})\right]}$ such that $\overline{Z\left[\mathcal{C}_{\mathcal{S B D}}(\mathcal{E})\right]}=$

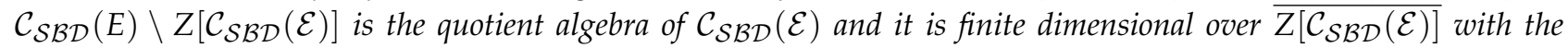
centre of $\mathcal{B}_{\mathcal{S B D}}=\overline{Z\left[\mathcal{C}_{\mathcal{S B D}}(\mathcal{E})\right]}$.

Proof. We know that $\mathcal{C}_{\mathcal{S B D}}(\mathcal{E})$ is prime and irreducible with its centre is non-zero by Proposition 4 . Since $\mathcal{B}_{\mathcal{S B D}}$ is simple from Lemma 1 , we can define $\mathcal{B}_{\mathcal{S B D}}=\left\{X W^{-1}, X \in \mathcal{C}_{\mathcal{S B D}}(\mathcal{E}) ; W \neq 0 \in\right.$ $Z\left[\mathcal{C}_{\mathcal{S B D}}(\mathcal{E})\right] \mid X_{1} W_{1}^{-1}=X_{2} W_{2}^{-1}$ if and only if $\left.X_{1} W_{2}=X_{2} W_{1}\right\}$. It follows that multiplication is closed under $\left(X_{1} W_{1}^{-1}\right)\left(X_{2} W_{2}^{-1}\right)=\left(X_{1} X_{2}\right)\left(W_{1} W_{2}\right)^{2}$ and addition $X_{1} W_{1}^{-1}+X_{2} W_{2}^{-1}=\left(X_{1} W_{1}+X_{2} W_{2}\right)\left(W_{1} W_{2}\right)^{-1}$. To show

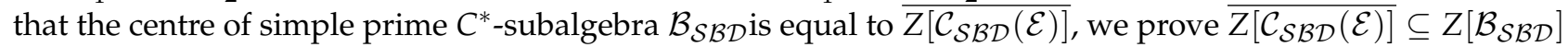

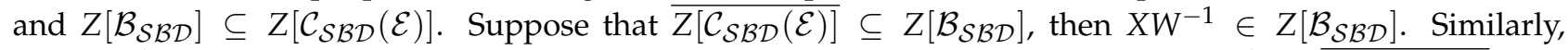

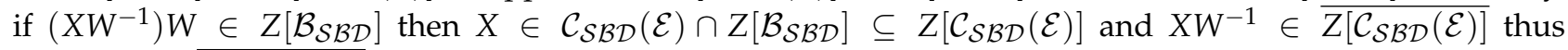
$Z\left[\mathcal{B}_{\mathcal{S B D}}\right]=\overline{Z\left[\mathcal{C}_{\mathcal{S B D}}(\mathcal{E})\right]}$. Furthermore, $\mathcal{B}_{\mathcal{S B D}}$ is prime since $A_{1} \mathcal{B}_{\mathcal{S B D}} A_{2}=0$ for all $A_{1}, A_{2} \in \mathcal{B}_{\mathcal{S B D}}$ and $A_{1}\left(X W^{-1}\right) A=0$ for all $X \in \mathcal{C}_{\mathcal{S B D}}(\mathcal{E}), W \neq 0 \in Z\left[\mathcal{C}_{\mathcal{S B D}}(\mathcal{E})\right]$. Suppose that $A_{1}=X_{1} W_{1}^{-1}, A_{2}=X_{2} W_{2}^{-1}$ for all $X_{i} \in \mathcal{C}_{\mathcal{S B D}}(\mathcal{E}), i=1,2$ and $W_{i} \in \mathcal{C}_{\mathcal{S B D}}(\mathcal{E}), i=1$, 2 . Then $0=X_{1} W_{1}^{-1} X W^{-1} X_{2} W_{2}^{-1}=\left(X_{1} X X_{2}\right)\left(W_{1} W W_{2}\right)^{-1}$ for all $X \in \mathcal{C}_{\mathcal{S B D}}(\mathcal{E}), W \in Z\left[\mathcal{C}_{\mathcal{S B D}}(\mathcal{E})\right]$. Hence, $X_{1} X X_{2}=0$ for all $X \in \mathcal{C}_{\mathcal{S B D}}(\mathcal{E})$. However, since $\mathcal{C}_{\mathcal{S B D}}(\mathcal{E})$ is prime, then either $X_{1}$ or $X_{2}$ is 0 and hence either $A_{1}$ or $A_{2}$ is 0 . Thus $\mathcal{B}_{\mathcal{S B D}}$ is prime and $\mathcal{B}_{\mathcal{S B D}}$ satisfies

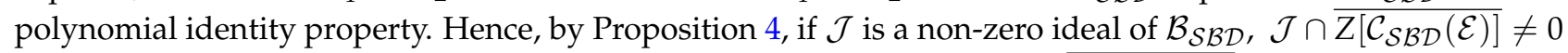
thus $\mathcal{J}=\mathcal{B}_{\mathcal{S B D}}$ and $\mathcal{B}_{\mathcal{S B D}}$ is simple and finite dimensional over its centre $\overline{Z\left[\mathcal{C}_{\mathcal{S B D}}(\mathcal{E})\right]}$.

Corollary 4. Let $\mathcal{C}_{\mathcal{S B D}}(\mathcal{E})$ be a unital $C^{*}$-subalgebra and $T=\sum_{i=1}^{n} A_{i} X B_{i}$ be an elementary operator on $\mathcal{C}_{\mathcal{S B D}}(\mathcal{E})$. If the elementary operator $T=\sum_{i=1}^{m} C_{i} X D_{i}$ where $C_{i} \in \mathbf{A}(T)$ and $D_{i} \in \mathbf{B}(T)$ for every $1 \leq i \leq m$. Then $\sum_{i=1}^{n} B_{i} A_{i}-$ $\sum_{i=1}^{m} D_{i} C_{i} \in Z\left[\mathcal{C}_{\mathcal{S B D}}(\mathcal{E})\right]$, where $\mathbf{A}(T)=\operatorname{span}\left\{A_{i}, \ldots, A_{n}\right\}$ the linear span of $A, \mathbf{B}(T)=\operatorname{span}\left\{B_{i}, \ldots, B_{n}\right\}$ the linear span of $B$ and $\mathbf{C}(T)=\operatorname{span}\left\{B_{i} A_{j} ; 1 \leq i, j \leq n\right\}$ the linear span of $B A$.

Proof. By Proposition 3 and Theorem $3, Z\left[\mathcal{C}_{\mathcal{S B D}}(\mathcal{E})\right]$ is a commutative $C^{*}$-subalgebra of $\mathcal{C}_{\mathcal{S B D}}(\mathcal{E})$ which is prime hence $\sum_{i=1}^{n} B_{i} A_{i}-\sum_{i=1}^{m} D_{i} C_{i} \in Z\left[\mathcal{C}_{\mathcal{S B D}}(\mathcal{E})\right]$. Let the length of $T$ be $n$ and $\left\{C_{1}, \ldots, C_{n}\right\}$ be linearly independent. If $n=m$, then $C_{i}=\sum_{k=1}^{n} \beta_{i k} A_{k}, 1 \leq i \leq n$ and $B_{k}=\sum_{k=1}^{n} \beta_{i k} D_{i}, 1 \leq k \leq n$. It follows that $\sum_{i=1}^{n} B_{i} A_{i}=\sum_{i=1}^{n} D_{i} C_{i}$. If $n<m$, then we express $C_{j}=\sum_{k=1}^{k} \beta_{j k} C_{k} \forall n+1 \leq j \leq m$ and the elementary 
operator $T=\sum_{i=1}^{n} C_{i} X D_{i}+\sum_{j=n+1}^{m} \sum_{k=1}^{n} \beta_{j k} C_{k} X D_{j}$. Thus $T=\sum_{i=1}^{n} C_{i} X D_{i}+\sum_{j=n+1}^{m} C_{k} X \beta_{j k} D_{j}$ which shows that $T=\sum_{i=1}^{n} C_{i} X D_{i}+\sum_{k=1}^{n} \sum_{j=n+1}^{m} C_{k} X \beta_{j k} D_{j}$, hence

$$
\begin{aligned}
T & =\sum_{k=1}^{n} C_{i} X D_{i}+\sum_{k=1}^{n} \sum_{j=n+1}^{m} C_{k} X \beta_{j k} D_{j} \\
& =\sum_{k=1}^{n} C_{k} X\left[D_{k}+\sum_{j=1}^{n} \beta_{j k} D_{j}\right] .
\end{aligned}
$$

Replacing $D_{k}^{0}=D_{k}+\sum_{j=1}^{n} \beta_{j k} D_{j}$, we have $\sum_{k=1}^{m} D_{k}^{0} C_{k}=\sum_{k=1}^{n} B_{k} A_{k}$. Thus, $\sum_{i=1}^{n} B_{i} A_{i}=\sum_{i=1}^{n} D_{i} C_{i}$ and $\sum_{i=1}^{n} B_{i} A_{i}-\sum_{i=1}^{m} D_{i} C_{i} \in Z\left[\mathcal{C}_{\mathcal{S B D}}(\mathcal{E})\right]$

Corollary 5. Let $\mathcal{A}_{\mathcal{S B D}}, \mathcal{B}_{\mathcal{S B D}}$ be two unital $C^{*}$-subalgebras of $\mathcal{C}_{\mathcal{S B D}}(E)$ and $\mathcal{I}$, $\mathcal{J}$ be ideals of $\mathcal{A}_{\mathcal{S B D}}$ and $\mathcal{B}_{\mathcal{S B D}}$ respectively. If $T: \mathcal{A}_{\mathcal{S B D}} \rightarrow \mathcal{B}_{\mathcal{S B D}}$ then there exists a spectrally bounded linear mapping $\widehat{T}: \mathcal{A}_{\mathcal{S B D}} / \mathcal{I} \rightarrow \mathcal{B}_{\mathcal{S B D}} / \mathcal{J}$ with $\|\widehat{T}\| \leq\|T\|_{\sigma}$ such that $B_{i} A_{i} \in Z\left[\mathcal{C}_{\mathcal{S B D}}(E)\right]$, for all $T=A_{i} X B_{i} \in \mathcal{C}(\mathcal{E})$.

Proof. Suppose that $X \in \mathcal{A}_{\mathcal{S B D}}$ and $Y \in \mathcal{I}$ such that the spectral radius of $r(X+Y) \leq r(X+I)+\varepsilon$, for any $\varepsilon>0$ then,

$$
\begin{aligned}
r(\widehat{T}(X+I)) & =r(T X+J) \leq \inf _{W \in \mathcal{J}} r(T X+W) \\
& =r(T X+T Y) \leq\|T\|_{\sigma} r(X+Y) \\
& =\|T\|_{\sigma}(r(X+\mathcal{I})+\varepsilon) .
\end{aligned}
$$

This means that $r(\widehat{T}(X+I)) \leq\|T\|_{\sigma} r(X+\mathcal{I})$ and $\|\widehat{T}\| \leq\|T\|_{\sigma}$ holds. Thus, by Corollary $4, B_{i} A_{i} \in$ $Z\left[\mathcal{C}_{\mathcal{S B D}}(E)\right]$.

Acknowledgments: The authors are grateful to the referees for the useful comments.

Author Contributions: All authors contributed equally to the writing of this paper. All authors read and approved the final manuscript.

Conflicts of Interest: "The authors declare no conflict of interest."

\section{References}

[1] Ara, P., \& Mathieu, M. (2002). Local Multiplier of $C^{*}$-algebras. Springer-verlag, London.

[2] Okelo, N. B., Ambogo, D. O., \& Nyakiti, S. A. (2010). On $C(\Omega)$ and $C_{S}(\Omega)$ of $C^{*}$-algebra and Norms of Derivations, International Mathematical Forum, 5(53), 2647-2653.

[3] Okelo, N. B., Okongo, M., \& Nyakiti, S. A. (2010). On Projective Tensor Norm and Norm-attainable $\alpha$-Derivations, International Journnal of Contemporary Mathematical Sciences, 5(40), 1969-1975.

[4] Okelo, N. B. (2018). Norm-attainability and Range-Kernel Orthogonality of Elementary Operators, Communications in Advanced Mathematical Sciences, 1(2), 91-98.

[5] Okelo, N. B. (2020). On Norm-attainable Operators in Banach Spaces, Journal of Function Spaces, 2020, 1-6.

[6] Shulman, V. S, \& Turovskii, Y. V. (2011). Topological Radicals II: Applications to Spectral Theory and Multiplication Operators, Springer, Basel.

[7] Nair, S. G. (2005). A Study of Elementary Operators, PhD Thesis, Cochin University of Science and Technology, Shangai, China.

[8] Boudi, N., \& Bracic, J. (2013). On Invertibility of Elementary Operators, arXiv:1312.1307.

[9] Hejazian S., \& Rostamani, M. (2010). Spectrally Compact Operators Tbilisi Mathematical Journal, 3, 17-25.

[10] Kittaneh, F. (2005). Spectral Inequalities for Hilbert Space Operators, Proceedings of American Mathematical Society, 134(2), 385-390.

[11] Okelo, N. B., \& Mogotu, P. O. (2019). Norm Inequalities and Orthogonality of commutators of Derivations, arxiv:190358v1.

[12] Mathieu, M. (1989). Elementary Operators on Prime $C^{*}$-algebras I, Mathematische Annalen, 284, $223-244$.

[13] Fong, K., \& Sourour, A. R. (1979). On the Operator Identity $\sum A_{k} X B_{k}=0$, Canadian Journal of Mathematics, 31(4), 845-857. 
[14] Gogic, I., \& Timoney, R. M. (2018). Closure of Two-sided Multiplications on $C^{*}$-algebra and Phantom Line Bundles, International Mathematics Research Notices, 2018(2), 607-640.

[15] Kumar, A., \& Rajpal, V. (2012). Symmetry and Quasi-centrality of Operator Space Projective Tensor Product, Archiv der Mathematik, 99, 519-529.

[16] Haily, A., Kaidi, A., \& Rodriguez-Palacios, A. (2011). Centralizers in Semisimple Algebras and Descent Spectrum in Banach algebras, Journal of Algebra, 347, 214-223.

[17] Bratteli, O. (1976). Centre of Approximate Finite-Dimensional C*-algebra, Journal of Functional Analysis, 21, $195-202$.

[18] Orhon, M. (2010). The Ideal centre of the Dual of Banach Algebra Lattice, Positivity, 14, 841-847.

[19] Sarsuor, J. \& As'ad, Y. A. (2001). Some Properties of Centrality in a Complex Banach Algebra, The Journal of Islamic University of Gaza, 9(2), 1-9.

[20] As'ad, Y. A. (2018). Extended Centerality in Complex Banach Algebra, International Mathematics Forum, 13(3), 117-122.

[21] Rennison, J. F. (1987). Conditions Related to Centrality in Banach Algebra II, Journal of London Mathematical Society, 2(35), 499-513.

[22] Kreyzig, E. (1978). Introductory Functional Analysis with Applications, John Wiley and sons, New York.

[23] Okelo, N. B., Agure, J. O., \& Ambogo, D. O. (2010). Norms of elementary operators and characterization of norm-attainable operators, International Journal of Mathematical Analysis, 24, 1197-1204.

[24] Conway, J. B. (2000). A Course in Operator Theory, American Mathematical Society, Rhode Island.

[25] Jung, Y. F.(1993). Spectrally Bounded Jordan Derivations on Banach Algebras, Bulletin of the Korean Mathematical Society, 36(3), 477-481.

[26] Palmer, T. W. (1984). Banach Algebras and the General Theory of*-algebras, Cambridge University Press, New York.

[27] As'ad, Y. A. (1989). Centrality in Banach Algebra, Masters Thesis, University of Jordan, Jordan.

[28] Mathieu, M., \& Schick, G. J. (2003). Spectrally Bounded Operators from Von Neumann Algebras, Journal of Operator Theory, 49, 285-293.

[29] Benard, A. (1980). Recent Trends in the Field of Jordan-Banach algebras, Functional Analysis and Operator Theory, Banach Centre Publications, Institute of Mathematics Polish Academy of Sciences Warszawa, 30, 9-19.

[30] Zimmer, R. J. (1990). Essentail Results of Functional Analysis, University of Chicago Press, Chicago.

[31] Morrison, T. J. (2001). Functional Analysis, An introduction to Banach Space Theory, A Wiley-interscience Publication, New York. 